\title{
polyCub: An R package for Integration over Polygons
}

\section{Sebastian Meyer $^{1}$}

1 Friedrich-Alexander-Universität Erlangen-Nürnberg (FAU), Erlangen, Germany

DOI: $10.21105 /$ joss. 01056

\section{Software}

- Review ¿

- Repository $\boldsymbol{c}^{\boldsymbol{1}}$

- Archive ¿

Submitted: 18 October 2018 Published: 12 February 2019

\section{License}

Authors of papers retain copyright and release the work under a Creative Commons Attribution 4.0 International License (CC-BY).

\section{Summary}

The integral of a continuously differentiable function $f(x, y)$ over a domain $W \subset \mathbb{R}^{2}$ can be approximated using an $n$-point cubature rule of the form

$$
\iint_{W} f(x, y) \mathrm{d} x \mathrm{~d} y \approx \sum_{i=1}^{n} w_{i} f\left(x_{i}, y_{i}\right),
$$

i.e., a weighted sum of function values at an appropriate set of nodes. In the special but common case of integration along the axes, i.e., $W=\left(x_{l}, x_{u}\right) \times\left(y_{l}, y_{u}\right)$, the domain is rectangular. Several software packages implement numerical integration over such rectangles (or hypercubes in higher dimensions), for example, the Cuba (Hahn, 2005) or cubature (Johnson, 2017) libraries, which are both interfaced from the cubature package (Narasimhan, Johnson, Hahn, Bouvier, \& Kiêu, 2018) in R (R Core Team, 2018).

In spatial statistics, however, the domains of interest typically correspond to geographic regions (administrative districts, lakes, etc.), which are described by polygons. Solving integrals over such complex domains requires specialized cubature methods, thus the $\mathrm{R}$ package polyCub. A simple graphical summary of the purpose of polyCub is given by its logo (see below).

polyCub implements the following methods for numerical integration over polygons:

- General-purpose product Gauss cubature (Sommariva \& Vianello, 2007)

- Simple two-dimensional midpoint rule via spatstat (Baddeley \& Turner, 2005)

- Adaptive cubature for radially symmetric functions $f(x, y)=f_{r}\left(\left\|\left(x-x_{0}, y-y_{0}\right)\right\|\right)$ via integration along the polygon boundary (Meyer \& Held, 2014, Supplement B)

- Accurate (but slow) integration of the bivariate Gaussian density based on polygon triangulation (Abramowitz \& Stegun, 1972, Section 26.9, Example 9)

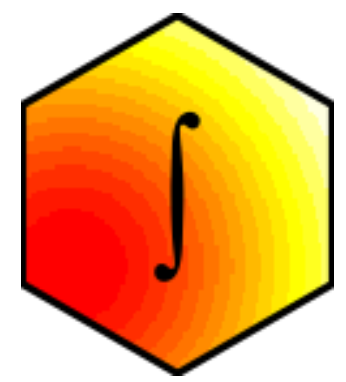

Figure 1: polyCub: cubature over polygonal domains. 


\section{Usage}

The $\mathrm{R}$ package polyCub is released on the Comprehensive R Archive Network (CRAN) and can thus be easily installed using install.packages ("polyCub") in R. After that, the basic usage is

library ("polyCub")

polyCub (polyregion, f)

where polyregion is the integration domain and $f$ is the integrand. Details are given in

vignette("polyCub")

which exemplifies the implemented cubature methods by solving the integral displayed in the package logo.

polyCub is currently used by at least two other R packages: in surveillance, to evaluate the likelihood of self-exciting spatio-temporal point process models for infectious disease spread (Meyer, Held, \& Höhle, 2017), and in rase, to integrate bivariate Gaussian densities for phylogeographic analyses (Quintero, Keil, Jetz, \& Crawford, 2015).

\section{References}

Abramowitz, M., \& Stegun, I. A. (Eds.). (1972). Handbook of Mathematical Functions with Formulas, Graphs, and Mathematical Tables. National bureau of standards applied mathematics series. New York: Dover Publications.

Baddeley, A., \& Turner, R. (2005). spatstat: An R package for analyzing spatial point patterns. Journal of Statistical Software, 12(6). doi:10.18637/jss.v012.i06

Hahn, T. (2005). Cuba - a library for multidimensional numerical integration. Computer Physics Communications, 168(2), 78-95. doi:10.1016/j.cpc.2005.01.010

Johnson, S. G. (2017). Adaptive multidimensional integration of a vector of integrands. Retrieved from https://github.com/stevengj/cubature

Meyer, S., \& Held, L. (2014). Power-law models for infectious disease spread. Annals of Applied Statistics, 8(3), 1612-1639. doi:10.1214/14-AOAS743

Meyer, S., Held, L., \& Höhle, M. (2017). Spatio-temporal analysis of epidemic phenomena using the R package surveillance. Journal of Statistical Software, 77(11). doi:10.18637/ jss.v077.i11

Narasimhan, B., Johnson, S. G., Hahn, T., Bouvier, A., \& Kiêu, K. (2018). cubatu re: Adaptive multivariate integration over hypercubes. Retrieved from https://CRAN. $\mathrm{R}$-project.org/package=cubature

Quintero, I., Keil, P., Jetz, W., \& Crawford, F. W. (2015). Historical biogeography using species geographical ranges. Systematic Biology, 64(6), 1059-1073. doi:10.1093/sysbio/ syv057

R Core Team. (2018). R: A language and environment for statistical computing. Vienna, Austria: R Foundation for Statistical Computing. Retrieved from https://www. R-project.org/

Sommariva, A., \& Vianello, M. (2007). Product Gauss cubature over polygons based on Green's integration formula. BIT Numerical Mathematics, 47(2), 441-453. doi:10.1007/ s10543-007-0131-2 